\title{
Estimating potential demand and supply of dengue vaccine in Brazil
}

\author{
Ananda Amarasinghe and Richard T. Mahoney* \\ International Vaccine Institute; Seoul, Korea
}

Key words: dengue, vaccines, markets, Brazil, immunization programs

\begin{abstract}
Dengue is endemic in Brazil. Several dengue vaccine candidates, including one at the Butantan Institute in Sao Paulo, are being evaluated in clinical trials and may be licensed in several years. This study estimates the potential doses of dengue vaccine needed in Brazil under different scenarios in the first 5 y after vaccine introduction. Estimates were based on 2015-2022 country population projections. An estimated country population of 200-209 million with an annual 3.3-3.5 million cohort in the 12 to 23 mo age group was included in the analysis. Computations were made for vaccines requiring 1,2 and 3 doses. A total of 7.8-62.9 million doses would be needed for only routine vaccination of $12-23$ mo cohort in first $5 \mathrm{y}$ with different vaccination schedules. A combination of country-wide routine 12-23-mo-old vaccination plus catchup vaccination of individuals up to $40 \mathrm{y}$ age is an appropriate strategy to control dengue. For this combination strategy, 129-425 million doses would be needed in the first 5 y after introduction. If vaccination is not provided to areas with low incidence of dengue, an estimated 108-360 million doses would be needed. This study provides a range of vaccine uptake estimates under different scenarios based on disease epidemiology. Actual demand and uptake will depend on the country vaccine introduction policy and strategies, vaccine supply capacity, cost and vaccine profile. We consider one option based on the availability of vaccine from different sources. A more advanced vaccine uptake model based on estimates of vaccine impact under various scenarios should be developed.
\end{abstract}

\section{Introduction}

Dengue virus (DENV) infection is a leading public health problem in Brazil. ${ }^{1}$ Available data indicate that dengue is endemic in most parts of the country. ${ }^{2,3}$ The disease is reported from all 5 regions and indigenous transmission has been reported in all but one of the 25 states. Brazil is responsible for over $60 \%$ of reported cases of dengue fever in the Americas region. ${ }^{4}$ In the past, the largest outbreak with a total of over 750,000 cases was reported in 2008. ${ }^{5}$ However, during the first 26 weeks in 2010, over 900,000 clinically suspected cases were reported, predicting that 2010 would be the worst. ${ }^{5}$ High incidences of Dengue Fever (DF) and Dengue Hemorrhagic Fever (DHF) are reported among young adults age of 20-40 y, but shifting of the disease into younger age groups has been observed in the past few years. ${ }^{2,3}$

Vector control is the only available measure of dengue prevention, but shows limited effectiveness and impact on disease control. ${ }^{6}$ There is no vaccine available to prevent dengue, but several dengue vaccine candidates are under development including one by Institute Butantan, a Brazilian public manufacturer, in collaboration with US NIH. ${ }^{78}$ If the planned clinical trials are successful, a dengue vaccine produced by Butantan might be available within the next 5-8 y. In addition, sanofi pasteur is developing a vaccine that is now in Phase $2 \mathrm{~b}$, and 3 trials and might achieve licensure in 2015 or earlier and could be introduced into Brazil.
The Oswaldo Cruz Foundation and GlaxoSmithKline are beginning collaboration on a purified inactivated vaccine. It is not possible to predict when this vaccine might be licensed. Based on epidemiological data and projections of likely uptake scenarios, we estimated the potential market size of dengue vaccine for Brazil in the first $5 \mathrm{y}$ after vaccine introduction and examine one illustrative scenario. We use the term "potential market size" to refer to projections of uptake by the national immunization program.

\section{Results}

Estimated target population for vaccination. According to census data and population predictions by the Instituto Brasileiro de Geografie e Estatistica (IBGE) 2008, the total projected population of Brazil will be around 200 million in 2015 and 209 million by 2022 (Table 1). The routine EPI serves the cohort of 12-23 mo age children which comprises a population of approximately 3.3-3.5 million children. The target population for catch-up immunization would range between 43.4-126.5 million with an upper age limit of $14 \mathrm{y}$ and $39 \mathrm{y}$ respectively

Routine immunization. For the dosing scenarios considered for the 12-23 mo age group, a total of 17.8 million to 62.9 million doses of dengue vaccine would be needed in the first $5 \mathrm{y}$ for the routine EPI to achieve full immunization (Table 2). 
Table 1. Estimated population (millions) for dengue vaccination in first 5 years of vaccine introduction

\begin{tabular}{|c|c|c|c|c|}
\hline & \multirow[b]{2}{*}{ Country population } & \multirow{2}{*}{$\begin{array}{l}\text { EPI Routine vaccination } \\
\text { 1-2 year (12-23 months) } \\
\text { cohort for } 5 \text { years }\end{array}$} & \multicolumn{2}{|c|}{ Mass vaccination } \\
\hline & & & $\begin{array}{c}\text { Option one: } 1-14 \text { years } \\
\text { cohort }\end{array}$ & $\begin{array}{c}\text { Option two: 1-39 years } \\
\text { cohort }\end{array}$ \\
\hline Population (million) & $200-209$ & $3.3-3.5$ & $37.6-43.4$ & $121.2-126.5$ \\
\hline As $\%$ of total population & 100 & $1.6-1.8$ & $17.9-21.7$ & $57.8-63.5$ \\
\hline $\begin{array}{l}\text { Population in selected regions } \\
\text { (millions) }\end{array}$ & $156-163$ & $2.2-2.4$ & $36.4-38.1$ & $100.7-105.2$ \\
\hline As $\%$ of total population & 78 & $1.1-1.2$ & $18.1-18.2$ & $50.2-50.3$ \\
\hline
\end{tabular}

Table 2. Total estimated vaccine volume (doses in million) in five years period of dengue vaccine introduction by different vaccination schedules and options

\begin{tabular}{|c|c|c|c|c|}
\hline & Option 1 & Option 2 & Option 3 & Option 4 \\
\hline & Only EPI (1-2 years) & $\begin{array}{c}\text { EPI (1-2 yrs) + Age 1-14 } \\
\text { yrs Cohort }\end{array}$ & $\begin{array}{c}\text { EPI (1-2 yrs) + Age 1-39 } \\
\text { year cohort }\end{array}$ & $\begin{array}{c}\text { EPI (1-2 yrs) + All ages } \\
\text { (>1 yrs) }\end{array}$ \\
\hline \multicolumn{5}{|l|}{ All country } \\
\hline Single dose schedule & $17.8-21.3$ & $54.8-65.6$ & $128.6-154.1$ & $186.6-228.0$ \\
\hline Two dose schedule & $34.9-41.9$ & $115.0-137.8$ & $250.8-300.6$ & $346.9-415.7$ \\
\hline Three dose schedule & $52.6-62.9$ & $194.3-202.6$ & $354.8-425.1$ & $493.2-590.9$ \\
\hline \multicolumn{5}{|l|}{ Selected Regions* } \\
\hline Single dose schedule & & $49.5-59.3$ & $108.4-133.3$ & 157.7-192.4 \\
\hline Two dose schedule & & $103.7-124.2$ & $212.1-254.1$ & $301.4-361.1$ \\
\hline Three dose schedule & & $152.5-182.7$ & $300.9-360.6$ & $421.0-504.5$ \\
\hline
\end{tabular}

Note: Ranges are for $10 \%$ and $25 \%$ vaccine wastage assumptions. Option 1, EPI only; Option 2, EPI+ 1-14 yrs cohort mass vaccination; Option 3, EPI + 1-39 yrs mass vaccination; Option 4, EPI $>1$ yrs all age groups. EPI, Country 1-2 age cohort for 5 years routine vaccination; Mass vaccination, one-time during 5 years.

The annual potential demand for routine vaccination of the 12-23 mo cohort is approximately 4 million doses of vaccine with a single dose vaccination schedule at $25 \%$ vaccine wastage. At the same wastage, for 2 and 3 doses schedules, it is around 8.4 and 12.5 million doses respectively. The declining birth rate will lead to a reduced total amount of vaccine needed, if vaccine introduction is delayed from 2015 to 2018. However this reduction is not significant for any vaccination schedules and does not exceed 2 million doses.

Catch-up vaccination. Dengue affects all age groups and therefore a vaccine may provide benefit at any age. To achieve a reduction of DENV transmission, a catch-up vaccination program (assuming the program begins by first immunizing the $12-23 \mathrm{mo}$ cohort) with broad age groups is necessary. The basic reproduction number $\left(\mathrm{R}_{\mathrm{o}}\right)$ estimated for dengue ranges between 1.33 and 11.6.9 In a study done in Sao Paulo State, $\mathrm{R}_{\mathrm{o}}$ is estimated as 2.7-11.6. ${ }^{10}$ Another multi-city study in Brazil estimated $\mathrm{R}_{\mathrm{o}}$ between 3.8 and 5.1. ${ }^{11}$ From the model of herd immunity developed by Ferguson and using an $\mathrm{R}_{\mathrm{o}}$ of 5.6, DENV transmission is predicted to stop at around $80 \%-85 \%$ immunity in the population. ${ }^{9,12}$ This must be interpreted to mean homotypic immunity in the case of circulation of a single type and tetravalent immunity where all four viruses are present. We do not know if 2 or 3 doses of a tetravalent dengue vaccine will give the same protection as 2 or 3 sequential natural DENV infections. Following a single administration of the monovalent candidate vaccines to sero-negative adult volunteers, the sero-conversion rates ranged from $46-100 \%$ for each DENV serotype. ${ }^{13}$ Dengue is endemic in Brazil and therefore it is expected that a significant proportion of the population has already seroconverted to more than one serotype. Therefore, for purposes of our calculations and for illustration purposes only, we assume that a single dose with very high tetravalent seroconversion rate given to $80 \%$ of the susceptible population could interrupt the DENV transmission. This further supports an assumption that there is no need for vaccination of the entire population to interrupt dengue transmission in the country. Based on these assumptions, we propose that the country should seek to provide the vaccine to people up to $40 \mathrm{y}$, which is around $60 \%$ of the population (Table 1 ). This will achieve an effective $80 \%$ level of immunity including those who are immune because of natural infection. These computations should be reviewed as data emerge from the use of tetravalent vaccines following licensure.

For a single-dose vaccine, demand would range between 36.6 million and 140.4 million doses for a catch-up vaccination strategy including 1-14 y old and 1-39 y old group over the initial 5-y period (Table 3). For a 2-dose scenario, the demand would range between 78.5 million and 267.1 million doses for the same age cohorts.

It is possible that the public will ask that all people receive the vaccine. For such a situation, the demand would total 167.5211.9 million doses for a one-dose vaccine and 442-540.6 million vaccine doses for a 3 -dose vaccine (Table 3 ).

Catch-up vaccination in priority regions. Some parts of Brazil have not been heavily affected by dengue. It is possible 
Table 3. Estimated vaccine volume (doses in million) for catch-up vaccination

\begin{tabular}{|c|c|c|c|c|c|c|}
\hline \multirow{2}{*}{$\begin{array}{c}\text { Catch-up } \\
\text { vaccination options } \\
\text { by different age } \\
\text { groups }\end{array}$} & \multicolumn{2}{|c|}{ Single dose schedule } & \multicolumn{2}{|c|}{ Two dose schedule } & \multicolumn{2}{|c|}{ Three dose schedule } \\
\hline & $10 \%$ Wastage & $25 \%$ Wastage & $10 \%$ Wastage & $25 \%$ Wastage & $10 \%$ Wastage & $25 \%$ Wastage \\
\hline \multicolumn{7}{|l|}{ All country } \\
\hline $1-14$ years Cohort & $36.6-40.6$ & $43.8-48.6$ & $78.5-87.1$ & $94.1-104.4$ & $114.5-127.1$ & $137.2-152.3$ \\
\hline $1-39$ years Cohort & $111.5-114.4$ & $137.1-140.4$ & $216.1-222.9$ & $259.0-267.1$ & $302.7-312.8$ & $362.7-374.8$ \\
\hline All age Cohort & $167.5-172.4$ & 200.7-211.9 & $319.0-326.5$ & $382.2-391.2$ & $442.0-451.2$ & $529.7-540.6$ \\
\hline \multicolumn{7}{|l|}{ Selected Regions } \\
\hline 1-14 years Cohort & $34.1-35.3$ & $40.8-42.3$ & $73.2-75.8$ & $87.7-90.8$ & $106.7-110.5$ & $127.9-132.4$ \\
\hline $1-39$ years Cohort & $91.2-94.2$ & $109.3-116.3$ & $178.3-184.2$ & $213.6-220.7$ & $250.7-258.9$ & $300.4-310.3$ \\
\hline All age Cohort & $139.0-143.5$ & $166.5-175.4$ & $264.9-273.5$ & $317.4-327.7$ & $367.1-379.0$ & $439.9-454.1$ \\
\hline
\end{tabular}

Note: Ranges are for lowest and highest estimate during 5 years due to varied annual population estimation.

that initially the government will focus on providing dengue vaccine to those regions that are most affected by dengue. The most affected regions are North East, North (only Amazon state), Central West (except Brasilia state) and South West (E. Luna, personal communication). We have computed the vaccine requirements for these regions only and made the same assumptions as we used for entire country estimates.

The total population of the more affected regions comprises about $78 \%$ of the country population (Table 1). An upper age limit of $39 \mathrm{y}$ for catch-up immunization in these regions would cover $50 \%$ of the whole country population. The total vaccine volume demand for mass vaccination in selected region would be $15-20 \%$ less than the entire country need. For single and 2 dose scenarios, the demand would range between 34.1 million and 220.7 million doses respectively for 1-14 y and 1-39 y cohorts vaccination options (Table 3). The highest demand with 3 dose schedules at $25 \%$ vaccine wastage for same 2 age groups would be 132.4 million and 310.3 million doses respectively.

Implications for local production and procurement. Various Brazilian government agencies are providing support for the development of dengue vaccines. Butantan Institute in Sao Paulo is working with the US. NIH to develop a live attenuated vaccine and the Oswaldo Cruz Foundation (FIOCRUZ) in Rio de Janeiro is now launching a collaboration with GlaxoSmithKline to develop a purified inactivated vaccine. The Butantan effort should lead to Phase 1 clinical trials in 2011 with licensure expected several years thereafter. The FIOCRUZ effort is somewhat farther upstream. The first priority of these producers will be to meet the national needs of Brazil. It will be important for both producers to understand the implications of potential vaccination strategies to design their production facilities to produce appropriate amounts. For purposes of this paper, considering vaccine availability, affordability and other logistics constraints, we consider one illustrative option for dengue vaccine introduction in 2015-2019 (Fig. 1). This option is based on a scenario that the sanofi pasteur vaccine will be licensed in Brazil in time for launch of immunization in 2015 and that the price of the sanofi pasteur vaccine may lead the government to limit delivery until such time as a local producer is licensed. We assume that a local producer will be licensed in time for immunization in 2017. Under these

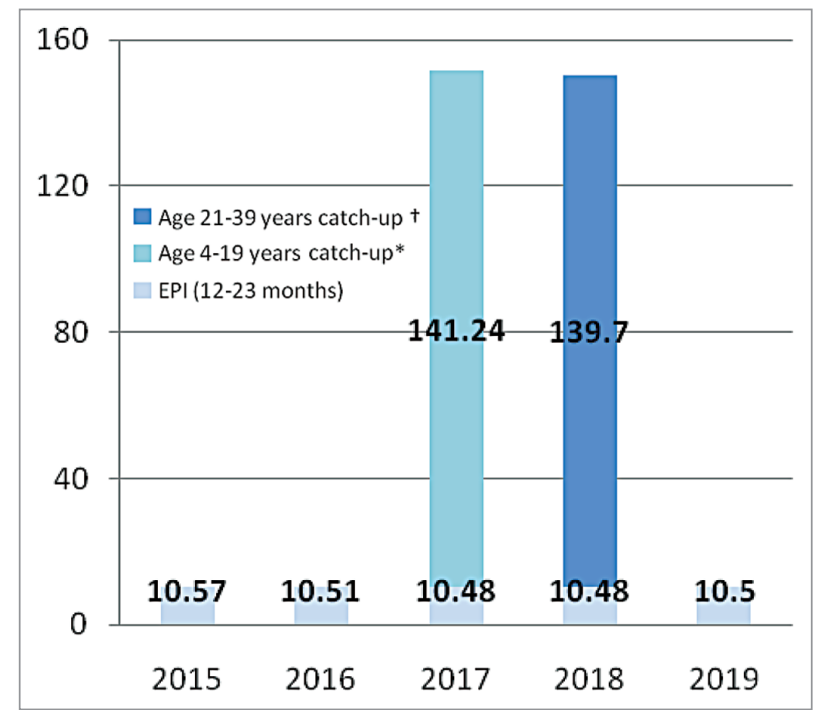

Figure 1. Vaccine introduction option for Brazil during 2015-2019: routine immunization of $12-23$ cohort and catch-up vaccination up to age of 39 years cohort with 3 doses schedule at $10 \%$ accine wastage (dengue vaccine doses in millions). *Children completed 2 and 3 years are already being vaccinated during 2015-6, (in routine EPI). They are excluded in catch-up vaccination in 2017. ${ }^{\dagger}$ Cohort in 20 years age in 2018 is excluded since they have been vaccinated in 2017 catch up program.

assumptions, we consider that Brazil will first start the routine immunization of 12-23 mo old cohort in 2015 and later implement and complete catch-up vaccination in the 2 y period, 20172018, covering a cohort of up to the age of $39 \mathrm{y}$. The scenario assumes a 3 -dose schedule at $10 \%$ vaccine wastage. Introducing routine immunization first has a few advantages, namely to monitor vaccine safety profile, plan for ensuring vaccine supply chain and prepare the logistic system for a catch-up vaccination program. With this option, annual need of dengue vaccine for routine immunization of $12-23$ mo age cohort is around 10.5 million doses. Catch-up vaccination will require additional 140 million doses in each year, during 2017-2018. The assumptions in these calculations are that it will be possible to administer a second vaccine in a population that has been receiving a first different vaccine. No clinical trials have been conducted to assess 
the feasibility of this option and thus the calculations made here may have to be revised based on future studies.

\section{Discussion}

Dengue is endemic in Brazil, and all four serotypes of DENV are reported in the country. Brazil has a strong disease surveillance system supported with a wide network of laboratories and an increasing prevalence of the disease has been observed in the last two decades. ${ }^{2,3}$ Dengue is reported among all age groups, but largely among the age group of less than $40 \mathrm{y}^{2,3}$ Despite a significant effort to control the disease, particularly through vector control, dengue represents an increasingly important public health challenge for the country. Dengue ranks as a top priority among health authorities. ${ }^{18}$

This paper makes estimates of the vaccine requirements of the government of Brazil during an initial 5-y period of a vaccination program launched in 2015 to 2019. We consider scenarios of nationwide immunization and a more limited program focusing on high priority regions. Ultimately decision making should rest on a number of additional considerations not considered here. For example, it will be useful to have a model that shows the impact of various vaccination strategies using the actual infection incidence levels of different age groups employing data from serosurveys. We have also not considered how demand would decline as catch-up immunization is completed.

Forecasting the demand years before the vaccine is licensed is difficult and has to be based on several assumptions including details of the vaccine product profile. The product profile will have a major impact on the vaccine uptake, particularly in relation to the number of doses required for protection. Since the number of doses required for full protection is not yet established, we adopted three possible scenarios from single dose to 2- or a 3-dose vaccination schedule. A long interval between doses will be a challenge to reaching high coverage, particularly for older age-groups in catch-up campaigns. Thus, we made our computations based on assumptions of age-specific drop-out rates. In addition, vaccine safety is of utmost important to reach the given estimates. Any unexpected adverse event(s) or fear of adverse effects due to lack of awareness would reduce the vaccine acceptance and thereby reaching the target population is unlikely as it has been reported with YF and influenza vaccination in Brazil. ${ }^{19,20}$ Another important factor influencing government policy will be the price of the vaccine which is currently unknown.

An important observation is that if the government waits on the availability of vaccine from one or more local producers before implementing catch-up immunization, the local producers will have to supply much more vaccine [ -150 million doses per year for $2 \mathrm{y}$ as shown in our illustrative option; (Fig. 1) than will be required for ongoing immunization ( -10 million doses)] following the completion of catch-up immunization. This raises the possibility that the local producers could become significant sources of vaccine for other countries following the completion of catch-up immunization in Brazil. This potential demand forecast could lead to building a plant that is too large (for domestic use) if the uptake is slow and then becomes inefficient (high cost of goods) if the doses cannot be sold elsewhere.

From the global dengue vaccine estimates by Pediatric Dengue Vaccine Initiative (PDVI), a total of 445-636 million doses of dengue vaccine represent upper-limit estimates of need in identified 20 early adopter countries. ${ }^{21}$ This upper-limit demand estimate for first $5 \mathrm{y}$ of vaccine introduction also included Brazil, but was computed for only up to $15 \mathrm{y}$ of age mass vaccination and routine EPI immunization of 1-2 y. When compared with the PDVI global estimates, the present study further supports the fact that Brazil has a potential high volume dengue vaccine need within a global vaccine scenario.

The difference observed between our estimates and the actual amount of vaccine used will depend on many other factors. Brazil may apply different vaccination strategies (routine or mass vaccination, geographic phasing or risk-group approach) and may reach different targets of performances (coverage and wastage). However we used the past performance data in Brazil for introducing new vaccines or conducting mass immunization activities, and believe that the differences observed will not be large. This paper illustrates the complexities faced by governments in the introduction of any new vaccine.

\section{Methods}

To estimate the potential need of vaccine in the first $5 \mathrm{y}$ after vaccine introduction in Brazil starting in 2015, we computed use for 1-, 2- and 3-dose regimens. The dengue vaccine product profile has not been fully established. The duration of protection conferred by a dengue vaccine is also unknown. Therefore, the need for a booster dose also is not clear. We assumed that even if a booster is necessary, it will not be required within $5 \mathrm{y}$ of completion of the primary course of vaccination. Hence, we excluded demand for any booster vaccination in this analysis.

Brazil has a strong national immunization program [NIP] and reported a high vaccine uptake of over $90 \%$ within a few years of new vaccine introduction into the NIP. ${ }^{14}$ Therefore, we estimate that the uptake for dengue vaccine in the first $5 \mathrm{y}$ after vaccine introduction would follow the same trend and reach a level between $90 \%$ and $95 \%$ of the target group. We used dropout rates (the rate at which individuals do not return for second or third doses) for routine vaccination of 12-23 mo age cohort, based on Expanded Program on Immunization (EPI) performances for hepatitis B vaccine which are less than $5 \%$ from first to third doses.

The reported coverage levels for mass vaccination among both children and adults with various antigens (influenza, meningococcal C vaccine, Yellow Fever) in Brazil are also high (70-95\%), and we used those coverage levels to estimate the coverage for dengue vaccination. ${ }^{15-17}$ Achieving high coverage with a 2 or 3-dose regimen and including older age-groups in the target population in mass vaccination pose challenges. Therefore, we assumed different mass vaccination coverage for different age cohorts. For the single-dose scenario, we assumed $90 \%$ coverage for the $12-23$ mo age group and $80 \%$ coverage for older age group. With 2 or 3 dose schedules, reaching high coverage in mass vaccination is 
difficult and unlikely. Therefore we set $10-30$ percentage point declines of coverage from the first to the second and from the second to the third doses for the older age groups, i.e., $>23$ mo. Brazil may launch immunization of the target populations all at once or in a phased manner.

The following formula was used to calculate the required number of vaccine doses for a given population: $\mathrm{n}=$ (target population) $\mathrm{x}$ (expected coverage) $\mathrm{x}$ (scheduled number of doses)/ (1 - wastage). For our analysis we used 10\% wastage for singledose vials and $25 \%$ wastage for multi-dose vials.

\section{References}

1. Camacho LAB. Yellow fever and public health in Brazil. Cad Saude Publica 2008; 24:482-3; DOI: 10.1590/ S0102-311X2008000300001.

2. Teixeira MG, Costa MN, Barreto F, Barreto ML. Dengue: twenty-five years since reemergence in Brazil. Cad. Saúde Pública 2009; 25:7-18; PubMed.

3. Teixeira MG, Costa MN, Barreto ML, Mota E Dengue and dengue hemorrhagic fever epidemics in Brazil: what research is needed based on trends, surveillance and control experiences? Cad Saude Publica 2005; 21:1307-15; DOI: 10.1590/S0102$311 \times 2005000500002$.

4. Nogueira RMR, Araujo JMG, Schatzmayr HG Dengue viruses in Brazil, 1986-2006. Rev Panam Salud Publica 2007; 22:358-63; DOI: 10.1590/S102049892007001000009.

5. PanAmerican Health Organization, World Health Organization; http://new.paho.org/hq/index. php?option=com_content\&task=view\&id 264\&Itemid $=363($ accessed on 20/Jul/2010).

6. Gratz NG, Halstead SB. The control of dengue vectors In: Halstead SB, editor. Dengue. London; Imperial College Press 2008; 5:361-87. (Pasvol G, Hoffman SL, Editors. Tropical medicine: science and practice; vol.5).

7. Webster DP, Farrar J, Rowland-Jones S. Progress towards a dengue vaccine. Lancet Infect Dis 2009; 9:678-87; DOI: 10.1016/S1473-3099(09)70254-3.

8. Souza J. Experimental dengue vaccine to be produced by 2010. The Bhutanta Institute. 08 June 2008; http://www.tropika.net/svc/news/20080608/ Souza20080608denguevacc.

\section{Acknowledgments}

This work was supported by a grant to the International Vaccine Institute from the Bill and Melinda Gates Foundation. (Grant No. 23197). We are indebted to Dr. Luiz Jacintho da Silva and Dr. Expedito Luna for their expert comments. We also acknowledge Dr. John Clemens, Director General, International Vaccine Institute for his valuable comments and editing this manuscript.
9. Halstead SB. Epidemiology. In: Halstead SB, editor. Dengue. London; Imperial College Press 2008; 5:75122. (Pasvol G, Hoffman SL, Editors. Tropical medicine: science and practice).

10. Massad E, Burattini MN, Coutinho FA, Lopez LF. Dengue and the risk of urban yellow fever reintroduction in Sao Paulo state, Brazil. Rev Saude Publica 2003; 37:477-84; DOI: 10.1590/S003489102003000400013

11. Favier C, Degallier N, Rosa-Freitas MG, Boulanger JP, Costa Lima JR, Luitgards-Moura JF, et al. Early determination of the reproductive number for vectorborne diseases: the case of dengue in Brazil. Trop Med Int Health 2006; 11:332-40; DOI: 10.1111/j.13653156.2006.01560.x.

12. Ferguson NM, Donnelly CA, Anderson RM. Transmission dynamics and epidemiology of dengue: insights from age-stratified sero prevalence surveys. Philos Trans R Soc Lond B Biol Sci 1999; 354:757-68 DOI: 10.1098/rstb.1999.0428.

13. Pediatric Dengue Vaccine Initiative, International Vaccine Institute. Policy makers' survey on dengue vaccine introduction 2009.

14. UNICEF-WHO. Immunization Summary: The 2009 edition [available from: http://www.childinfo.org/files/ Immunization_Summary_2009.pdf].

15. da Cunha SS, Camacho LAB, Santosa AC, Dourado I. Influenza vaccination in Brazil: rationale and caveats. Rev Saude Publica 2005; 39:129-36; DOI: $10.1590 /$ S0034-89102005000100017.
16. Kupek E, Puricelli RCB, Westrupp MHB. Effectiveness of a mass immunization campaign against serogroup $\mathrm{C}$ meningococci in children in the Federal State of Santa Catarina, Brazil. Braz J Infect Dis 2001; 5:324-31; DOI: $10.1590 / S 1413-86702001000600006$

17. Yellow Fever vaccination in Brazil 1997-2004. Ministry of Health, Brazil available from: http://portal.saude. gov.br/portal/arquivos/pdf/FA\%20TOTAL $\% 20 \mathrm{E} \% 20$ END\%20UF\%20MEN\%2015\%20E\%20MAI\%20 97\%20A\%202004\%20FINAL\%20REVISADO \%20F. pdf (in Portuguese).

18. Whitehead SS, Blaney JE, Durbin AP, Murphy BR Prospects for a dengue virus vaccine. Nat Rev Microbiol 2007; 5:518-28; DOI: 10.1038/nrmicro1690.

19. Tauil PL. Critical aspects of yellow fever control in Brazil. Rev Saude Publica 2010; 44:555-8; DOI: 10.1590/S0034-89102010005000014.

20. Dip RM, Cabrera MAS. Influenza vaccination in noninstitutionalized elderly: a population-based study in a medium-sized city in Southern Brazil. Cad. Saúde Pública, Rio de Janeiro 2010; 26:1035-44.

21. Amarasinghe A, Wichmann O, Margolis HS, Mahoney RT. Forecasting dengue vaccine demand in disease endemic and non-endemic countries. Hum Vaccin 2010; 6:745-53; DOI: 10.4161/hv.6.9.12587. 